\title{
Voltage- and Light-Controlled Spin Properties of a Two-Dimensional Hole Gas in p-Type GaAs/AIAs Resonant Tunneling Diodes
}

\author{
H.V.A. GALETI, ${ }^{1,5}$ Y. GALVÃO GOBATO ${ }^{2}$ M.J.S.P. BRASIL ${ }^{3}$ D. TAYLOR, ${ }^{4}$ \\ and M. HENINI ${ }^{4}$ \\ 1.-Departamento de Engenharia Elétrica, Universidade Federal de São Carlos, São Carlos 13560-905, \\ Brazil. 2.-Departamento de Física, Universidade Federal de São Carlos, São Carlos 13560-905, Brazil. \\ 3.-Instituto de Física "Gleb Wataghin", UNICAMP, Campinas 13083-859, Brazil. 4.-School of Phy- \\ sics and Astronomy, Nottingham Nanotechnology and Nanoscience Centre, University of Nottingham, \\ Nottingham NG7 2RD, UK. 5.-e-mail: helder@ufscar.br
}

We have investigated the spin properties of a two-dimensional hole gas (2DHG) formed at the contact layer of a $p$-type GaAs/AlAs resonant tunneling diode (RTD). We have measured the polarized-resolved photoluminescence of the RTD as a function of bias voltage, laser intensity and external magnetic field up to $15 \mathrm{~T}$. By tuning the voltage and the laser intensity, we are able to change the spin-splitting from the $2 \mathrm{DHG}$ from almost $0 \mathrm{meV}$ to $5 \mathrm{meV}$ and its polarization degree from $-40 \%$ to $+50 \%$ at $15 \mathrm{~T}$. These results are attributed to changes of the local electric field applied to the two-dimensional gas which affects the valence band and the hole Rashba spin-orbit effect.

Key words: Two-dimensional hole gas, spintronics, resonant tunneling diodes, photoluminescence

\section{INTRODUCTION}

The investigation of two-dimensional hole gases (2DHGs) in semiconductor nanostructures has been the subject of research in recent years for fundamental physics and possible applications in spintronic devices. ${ }^{1-9}$ It is well known that the $2 \mathrm{DHG}$ dispersion is characterized by a strong spin-orbit interaction, which can lead to spin-splitting of the bands even in the absence of an external magnetic field. This effect makes such systems very interesting for spintronic applications. ${ }^{1}$ In addition, wavefunctions of holes have little overlap with the nuclei which can improve the spin coherence time, rendering the spin of holes a promising candidate for applications in spintronics. For instance, it has been reported that localized hole spins in $p$-doped III-V semiconductors can have considerably long spin lifetimes in the range of $100 \mu \mathrm{s}$ and coherence times

(Received October 30, 2017; accepted January 4, 2018; published online January 16, 2018) of the order of $\mu$ s which are useful for spintronics. ${ }^{10,11}$ Furthermore, resonant tunneling diodes (RTDs) are interesting devices for studying the physical properties of $2 \mathrm{D}$ systems due to the fact that their carrier density and the resulting parameters, such as the filling factor, can be varied by the applied bias voltage. ${ }^{12-21}$ Several previous works have shown that $2 \mathrm{DHG}$ formed in the accumulation layer can inject spin-polarized holes in the quantum well $(\mathrm{QW})$ region and make an important contribution to the spin polarization of carriers in resonant tunneling diodes. ${ }^{21}$

In this paper, we have investigated the effect of the electric field and laser excitation intensity on the spin properties of $2 \mathrm{DHGs}$ formed at the accumulation layer next to the emitter barrier of a $p$ type GaAs/AlAs resonant tunneling diode. We studied the current-voltage $[\mathrm{I}(\mathrm{V})]$ characteristics and the polarization-resolved photoluminescence (PL) as a function of applied voltage and laser intensity under high magnetic fields (up to 15T) parallel to the tunnel current. Our results show evidence of a spin-polarized 2DHG emission formed at the 
accumulation layer next to the emitter barrier in high magnetic fields up to $15 \mathrm{~T}$. The $2 \mathrm{DHG}$ spinsplitting and polarization degree are voltage- and light-sensitive. In particular, the 2DHG polarization exhibits strong oscillations near hole resonances and seems to follow the voltage dependence of the excitonic spin-splitting. Our experimental results show evidence of electric field coupling to the hole spin degree of freedom for the spin orbit and Stark effects in the hole electronic structure.

\section{EXPERIMENTAL}

The samples investigated in this work are p-i-p resonant tunneling diodes grown by Molecular Beam Epitaxy on $\mathrm{n}+(001)$ GaAs substrate. The layer structure consists of $3 \mu \mathrm{m}$ p-GaAs $\left(2 \times 10^{18} \mathrm{~cm}^{-3}\right), 0.1 \mu \mathrm{m} \mathrm{p}$-GaAs $\left(10^{18} \mathrm{~cm}^{-3}\right), 0.1 \mu \mathrm{m}$ p-GaAs $\left(5 \times 10^{17} \mathrm{~cm}^{-3}\right), 51 \AA$ undoped GaAs spacer, $51 \AA$ AlAs barrier, $51 \AA$ GaAs QW, $51 \AA$ AlAs barrier, $51 \AA$ GaAs spacer, $0.1 \mu \mathrm{m} \mathrm{p}$-GaAs $\left(5 \times 10^{17}\right.$ $\mathrm{cm}^{-3}$ ), and $0.1 \mu \mathrm{m} \mathrm{p}-\mathrm{GaAs}\left(10^{18} \mathrm{~cm}^{-3}\right)$, and $0.6 \mu \mathrm{m}$ $\mathrm{p}$-GaAs $\left(2 \times 10^{18} \mathrm{~cm}^{-3}\right)$. The samples were processed with annular AuGe contacts with optical mesas of $400 \mu \mathrm{m}$ diameter. Polarization-resolved magneto-photoluminescence measurements were performed at $4 \mathrm{~K}$ using an Oxford magnet with an optical window under magnetic fields up to $15 \mathrm{~T}$ and a Si CCD system coupled to a $0.50-\mathrm{m}$ Andor spectrometer. A green solid state laser $(532 \mathrm{~nm})$ was used for light excitation. Circularly polarized emission was selected by using appropriate optics.

\section{RESULTS AND DISCUSSION}

Figure 1a shows a schematic diagram of the potential profile of the structure. Under applied bias, holes are accumulated next to the barriers forming a 2DHG. Resonant tunneling condition is obtained when the energy of $2 \mathrm{D}$ confined energy states at the accumulation layer are equal to a $\mathrm{QW}$ resonant energy level (labeled HH1, LH1, HH2 and LH2). Under light excitation, electrons are photogenerated in the contact region and optical recombination can occur in different regions of the device under applied bias, as illustrated in Fig. 1a. Figure $1 \mathrm{~b}$ shows typical polarization-resolved PL spectra for a $0.30-\mathrm{V}$ bias voltage without a magnetic field. The emission at $\sim 1.64 \mathrm{eV}$ is attributed to recombination from the fundamental state of the QW (E1-HH1). The emission from the GaAs contact layers includes two main bands: a broad band at $\sim 1.49 \mathrm{eV}$ associated to recombination between free electrons and holes bound to acceptors in the degenerated $p$-doped layers (labeled e-A) and a weaker band at $\sim 1.46 \mathrm{eV}$ which we attribute to the spatially indirect recombination between holes from the 2DHG at the accumulation layer and free tunneling electrons (labeled e-2DHG), as will be discussed later. In the following, we will focus on the spin properties of the holes from the $2 \mathrm{DHG}$ in the accumulation layer.

Figure 1c displays the $\mathrm{I}(\mathrm{V})$ curves under light excitation and dark conditions. Several structures are observed which we associate with resonant tunneling conditions through the $\mathrm{HH} 1, \mathrm{LH} 1, \mathrm{HH} 2$, and HH3 QW valence band levels, as identified in Fig. 1a. An additional structure is also observed at $0.5 \mathrm{~V}$ bias, solely under light excitation, which was associated to resonant tunneling of photo-generated electrons through the E1 QW state.

Figure 2a presents color-coded maps of the PL emission intensity associated to the GaAs contact layers as a function of bias voltage for $B=0 \mathrm{~T}$ under laser excitation $\left(3.5 \mathrm{~W} / \mathrm{cm}^{2}\right)$. The $\mathrm{I}(\mathrm{V})$ curve is also shown in order to analyze possible correlations between the optical emission and the transport characteristics. We have observed that the PL of the GaAs contact layer is dominated by the broad band from the recombination of electrons and holes of degenerated $p$-doped GaAs layers at $\sim 1.49 \mathrm{eV}$. As expected, this contact emission remains basically constant, independent of the applied bias voltage. In contrast, the weaker band at smaller energy is critically voltage-sensitive. This band emerges from the $1.49 \mathrm{eV}$ main band at $\sim 0.1 \mathrm{~V}$, and shows a strong red shift with increasing applied bias voltages. For large voltages, the intensity of this band decreases and it becomes much weaker for biases $>0.45 \mathrm{~V}$. This behavior is fully consistent with the optical recombination between tunneling electrons and holes from the 2DHG formed at the accumulation layer (e-2DHG emission). We have also analyzed the voltage dependence of the e-2DHG recombination in detail by subtracting the constant PL emission from the degenerated $p$-doped GaAs layers (Fig. 2b). For increasing bias voltages, the electric field increases along the structure and the band bending results in an energy shift of the e2DHG transition to smaller energies. Furthermore, the larger electric field gives rise to an increasing spatial separation between the electrons and the holes. Therefore, a decreasing overlap of the carriers' wavefunctions is expected, and, subsequently, a decreasing PL intensity with increasing bias.

Under light excitation and applied bias voltage, both two-dimensional electron (2DEG) and hole (2DHG) gases should be formed at the accumulation layers next to the barriers. ${ }^{20}$ We attribute the observed low-energy peak to the e-2DHG recombination based on previous reported experimental results that showed that optical transitions involving a 2DHG are observed both with and without an external magnetic field, while transitions associated to the 2DEG are observed solely in the presence of external magnetic fields. ${ }^{20}$ The distinct behavior of 2DEG and 2DHG emissions must be associated to different effective masses and mobility of electrons and holes. 

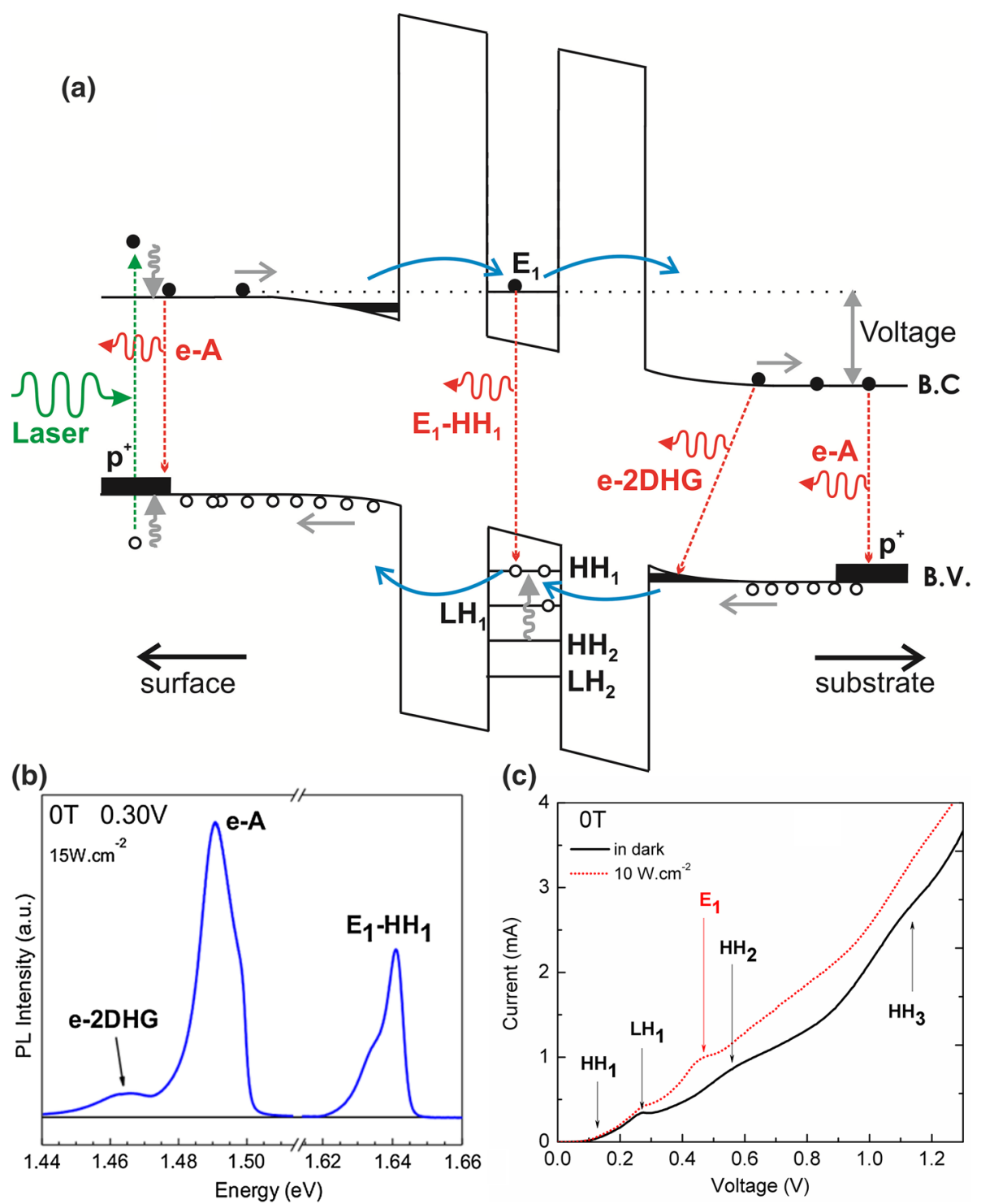

Fig. 1. (a) Schematic potential profile of the sample layer structure; (b) typical PL spectra; (c) I(V) characteristic curves under dark and light excitation.
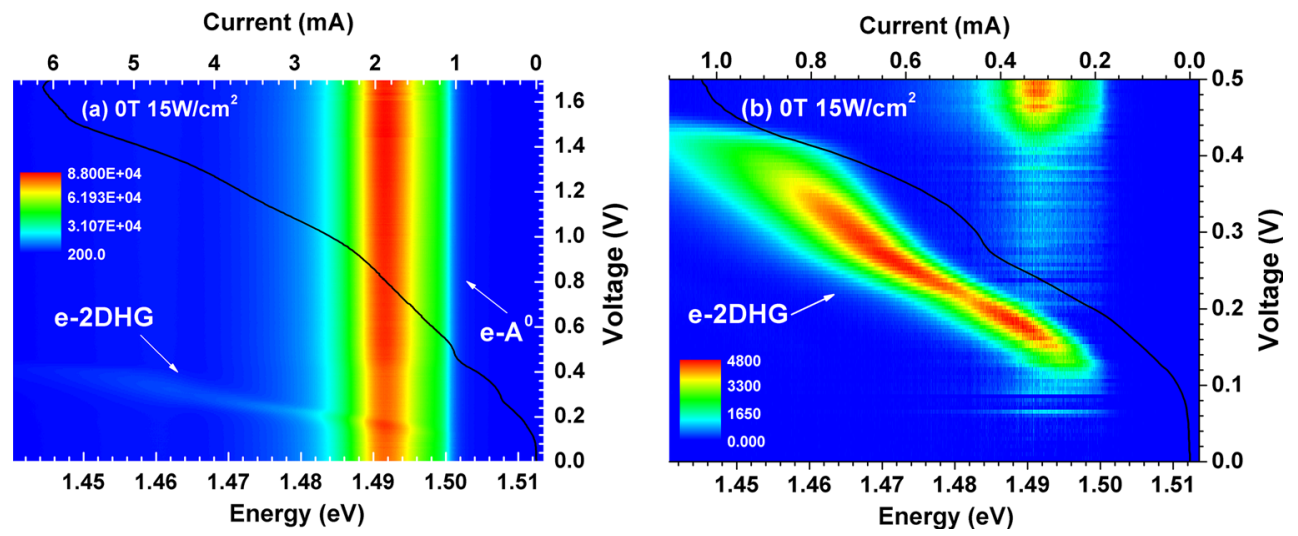

Fig. 2. (a) and (b) Color-coded maps of the RTD GaAs contact emission as a function of applied bias voltage under $B=0 \mathrm{~T}$ (Color figure online). 
Figure $3 \mathrm{a}$ and $\mathrm{b}$ presents the color-coded maps of the contact emission for right $(\sigma+)$ and left $(\sigma-)$ circularly polarized emission at $15 \mathrm{~T}$. In order to analyze the contribution due to the $2 \mathrm{DHG}$, we have also subtracted the emission from the contact layers. Figure $3 c$ and d was obtained by subtracting the constant emission from the GaAs contact layers. It is worth pointing out that the $2 \mathrm{DHG}$ emission is clearly $\sigma$ - circularly polarized and voltage-dependent. On the other hand, the GaAs broad band is $\sigma+$ circularly polarized. We have estimated the polarization degree of the GaAs broad band to be $\sim+10 \%$ at $15 \mathrm{~T}$ which is independent of the applied bias voltage.

Figure 4 illustrates the bias voltage dependence of (a) the current, (b) the total PL integrated intensity, (c) the spin-splitting energy and (d) the degree of circular polarization (DCP) for the e2DHG emission band at 15T for two laser excitation intensity conditions, $3.5 \mathrm{~W} / \mathrm{cm}^{2}$ and $15 \mathrm{~W} / \mathrm{cm}^{2}$. As discussed before, the intensity of the e-2DHG emission band increases for increasing voltages up to $\sim 0.3 \mathrm{~V}$, reflecting the increasing density of the 2DHG with applied bias. For larger voltages, the strong electric field separates the electrons and holes, and the intensity of the spatially indirect e2DHG emission tends to vanish. However, we observe from Fig. 4b that the e-2DHG intensity shows oscillations with increasing applied bias, which must be related to the resonant tunneling of holes through the double barrier. Even though the peaks in the $\mathrm{I}(\mathrm{V})$ curves are not clearly resolved, the $\mathrm{PL}$ intensity minimum at $\sim 0.24 \mathrm{~V}$ must be related to the onset of a hole resonance.

In addition, we observed that the e-2DHG spinsplitting energy is very sensitive to the laser intensity and applied voltage. In particular, an increase of the spin-splitting is observed by increasing the laser intensity for voltages $>0.18 \mathrm{~V}$. For the lower excitation intensity, the e-2DHG spin-splitting is rather small and tends toward negative values, except for low bias voltages $(<0.2 \mathrm{~V})$ when it becomes positive with a value of $\sim 1 \mathrm{meV}$. For higher excitation intensity, the spin-splitting becomes significantly larger and shows some oscillations, but it tends to increase with applied bias up to $\sim 5 \mathrm{meV}$. The voltage and the light intensity dependence of the spin-splitting energy must be associated to significant variations of the $2 \mathrm{DHG}$ density and to the local electric field, which couples the spin degree of freedom of the confined holes.

We have also calculated the DCP of the e-2DHG emission by using the relationship: $\left(I^{\sigma+}-I^{\sigma-}\right) /$ $\left(I^{\sigma+}+I^{\sigma-}\right)$, where $I^{\sigma+}$ and $I^{\sigma-}$ are the integrated PL
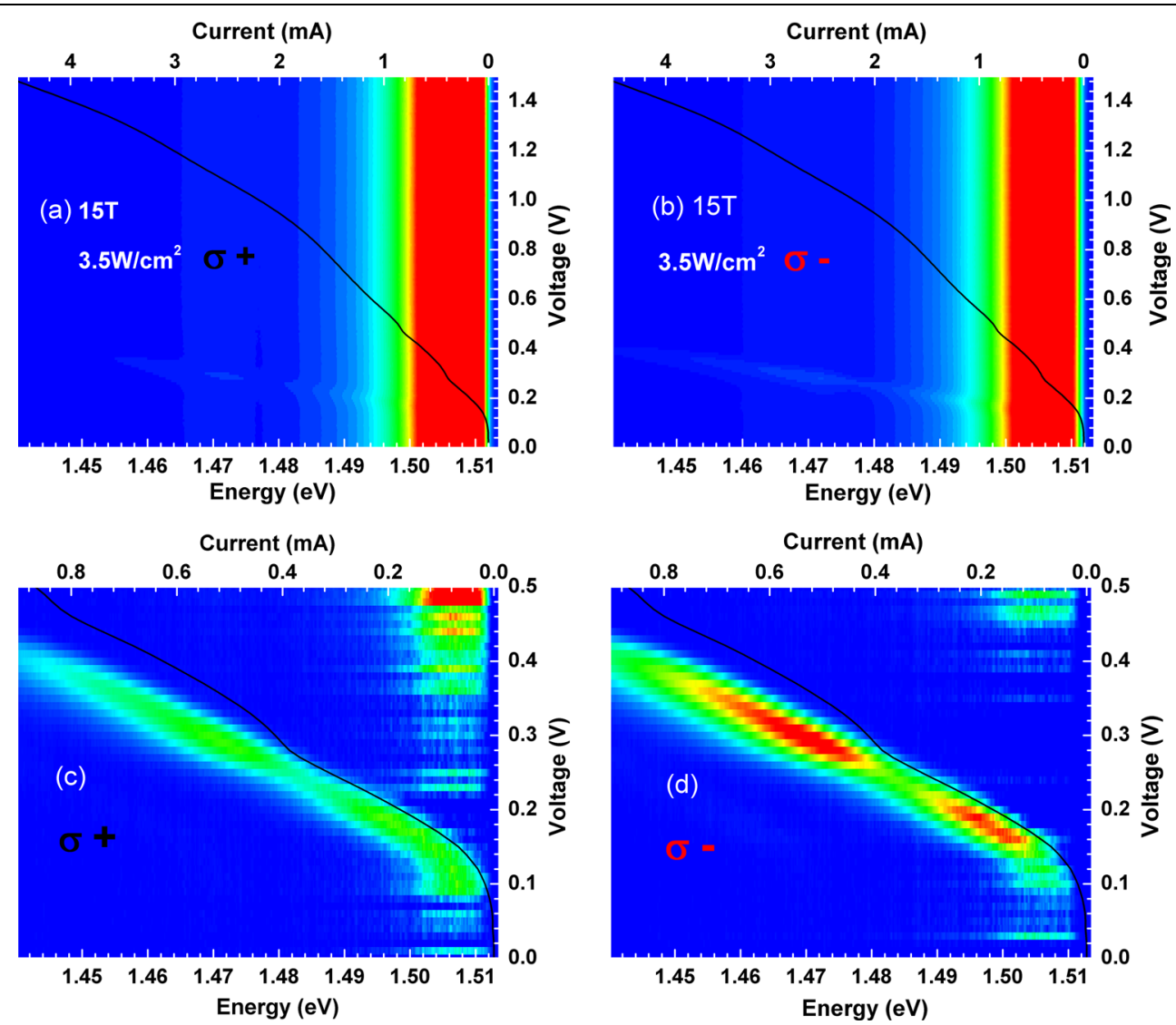

Fig. 3. (a)-(d) Color-coded maps of the circularly polarized contact emissions as a function of applied bias voltage under $B=15 \mathrm{~T}$ (Color figure online). 


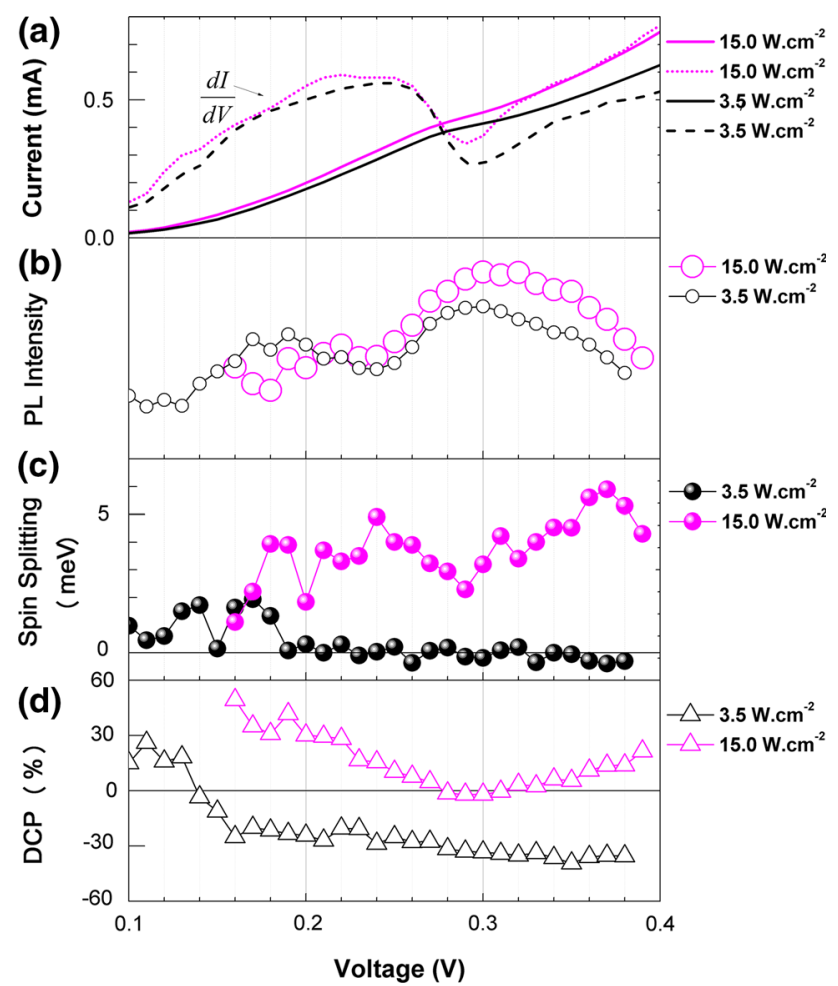

Fig. 4. (a) $\mathrm{I}(\mathrm{V})$ characteristics curves and $\mathrm{d} I / \mathrm{d} V$ (dashed lines); (b) total PL intensity; (c) spin-splitting, and (d) polarization degree of the contact layer emission as a function of applied voltage and for two different laser intensities.

intensities of the right and left circularly polarized emissions. We have observed that both the spinsplitting and the circularly polarization degree of e2DHG emissions are very sensitive to laser power and applied voltage. In addition, they show sign inversion at different laser intensity conditions. Actually, for applied voltages $>0.15 \mathrm{~V}$, the polarization degree has a positive value for the higher laser intensity and a negative value for the lower laser intensity. Furthermore, we note that, for the lower excitation intensity condition, the 2DHG-e polarization degree shows a signal inversion around $0.15 \mathrm{~V}$. The sign of the polarization degree seems to be correlated to the sign of the Zeeman splitting of the 2DHG (Fig. 4c). In fact, the Zeeman splitting is positive for the higher laser intensity and has a tendency to be negative for low laser power and higher voltages. The physical origin of this sign inversion and the voltage dependence of the DCP and spin-splitting are difficult to interpret quantitatively, and a theoretical model would be necessary in order to understand the obtained results. However, our experimental results must be attributed to the electric field-induced coupling of the hole spin degree of freedom, the Rashba spin orbit and Stark effects on the whole electronic structure. ${ }^{15}$

\section{CONCLUSION}

In summary, we have investigated the spatially indirect emission 2DHG-e involving spin-polarized hole gas formed at the accumulation layers of resonant tunneling diodes under high magnetic fields. We have observed that the spin splitting and the polarization degree of the 2DHG emission are voltage- and light-dependent. In particular they show sign inversion for different laser power conditions. These results, which are attributed to the effects of changes of the electric field in the valence band of the RTD and the modulation of hole Rashba spin-orbit, show the importance of valence band contributions for the spin polarization of carriers in $p$-type resonant tunneling diodes. These findings can be useful for spintronic devices whose operation relies on the tuning of the spin splitting by applying an electric field, and should also stimulate further experimental and theoretical investigations of the spin properties of semiconductor heterostructures and devices.

\section{ACKNOWLEDGEMENTS}

The financial supports from the Brazilian Agencies FAPESP (Grants: 16/10668-7 and 12/24055-6) and $\mathrm{CNPq}$ and from the UK Engineering and Physical Sciences Research Council, are gratefully acknowledged.

\section{REFERENCES}

1. R. Winkler, Spin-Orbit Coupling Effects in Two-Dimensional Electron and Hole Systems (Berlin: Springer, 2003), pp. 1-6.

2. J.P. Lu, J.B. Yau, S.P. Shukla, M. Shayegan, and L. Wissinger, Phys. Rev. Lett. 81, 1282 (1998).

3. J.P. Lu, M. Shayegan, L. Wissinger, U. R̈ossler, and R. Winkler, Phys. Rev. B 60, 13776 (1999).

4. L.P. Rokhinson, V. Larkina, Y.B. Lyanda-Geller, L.N. Pfeiffer, and K.W. West, Phys. Rev. Lett. 93, 146601 (2004).

5. M. Studer, M. Hirmer, D. Schuh, W. Wegscheider, K. Ensslin, and G. Salis, Phys. Rev. B 84, 085328 (2011).

6. M. Kugler, K. Korzekwa, P. Machnikowski, C. Gradl, S. Furthmeier, M. Griesbeck, M. Hirmer, D. Schuh, W. Wegscheider, T. Kuhn, C. Schuller, and T. Korn, Phys. Rev. $B$ 84, 085327 (2011).

7. D. Kamburov, M. Shayegan, R. Winkler, L.N. Pfeiffer, K.W. West, and K.W. Baldwin, Phys. Rev. B 86, 241302 (2012).

8. M. Kugler, T. Andlauer, T. Korn, A. Wagner, S. Fehringer, R. Schulz, M. Kubová, C. Gerl, D. Schuh, W. Wegscheider, P. Vogl, and C. Schüller, Phys. Rev. B 80, 035325 (2009).

9. B. Habib, E. Tutuc, S. Melinte, M. Shayegan, D. Wasserman, S.A. Lyon, and R. Winkler, Phys. Rev. B 69, 113311 (2004).

10. C. Damen, L. Vina, J.E. Cunningham, J.E. Shah, and L.J. Sham, Phys. Rev. Lett. 67, 3432 (1991).

11. P. Schneider, J. Kainz, S.D. Ganichev, V.V. Bel'kov, S.N. Danilov, M.M. Glazov, L.E. Golub, U. Rössler, W. WegScheider, D. Weiss, D. Schuh, and W. Prettl, J. Appl. Phys. 96, 420 (2004).

12. A. Slobodskyy, C. Gould, T. Slobodskyy, C.R. Becker, G. Schmidt, and L.W. Molenkamp, Phys. Rev. Lett. 90, 246601 (2003).

13. M.M. Glazov, P.S. Alekseev, O.M. Adnoblyudov, V.M. Chistyakov, S.A. Tarasenko, and I.N. Yassievich, Phys. Rev. $B$ 71, 155313 (2005). 
14. J. Fabian, A. Matos-Abiague, C. Ertler, P. Stano, and I. Zutic, Acta Physica Slovaca 57, 565 (2007).

15. H.B. de Carvalho, M.J.S.P. Brasil, V. Lopez-Richard, Y. Galvão Gobato, G.E. Marques, I. Camps, L.C.O. Dacal, M. Henini, L. Eaves, and G. Hill, Phys. Rev. B 74, 041305 (2006).

16. H.B. de Carvalho, M.J.S.P. Brasil, Y. Galvão Gobato, G.E. Marques, H.V.A. Galeti, M. Henini, and G. Hill, Appl. Phys. Lett. 90, 62120 (2007).

17. L.F. dos Santos, Y. Galvão Gobato, G.E. Marques, M.J.S.P. Brasil, M. Henini, and R. Airey, Appl. Phys. Lett. 91, 073520 (2007)
18. L.F. dos Santos, Y. Galvão Gobato, G.E. Marques, M.J.S.P. Brasil, M. Henini, and R. Airey, Appl. Phys. Lett. 92, 143505 (2008).

19. Y.G. Gobato, J.M. Berroir, Y. Guldner, J.P. Vieren, F. Chevoir, and B. Vinter, Phys. Rev. B. 44, 13795 (1991).

20. A. Vercik, Y. Galvão Gobato, and M.J.S.P. Brasil, J. Appl. Phys. 92, 1888 (2002).

21. Y. Galvão Gobato, H.V.A. Galeti, L.F. dos Santos, V. LópezRichard, D.F. Cesar, G.E. Marques, M.J.S.P. Brasil, M. Orlita, J. Kunc, D.K. Maude, M. Henini, and R.J. Airey, Appl. Phys. Lett. 99, 233507 (2011). 